\title{
Development of an accurate nursing program based on function in chronic venous leg ulcers: protocol for matching function status with nursing interventions using the Delphi survey ${ }^{\dagger}$
}

\author{
Original article \\ Yi-Fan Tang ${ }^{\mathrm{a}}$, Xin-Yu Hü, Jing Zhaob, Ze-Juan Guc,*\%, Shuang-Shuang Xinga, Wei-Yun Wang ${ }^{\mathrm{a}}$ \\ aCollege of Nursing, Nanjing Medical University, Naniing, Jiangsu 211166, China \\ ${ }^{b}$ Department of Outpatient Treatment, The First Affiliated Hospital with Nanjing Medical University, Nanjing, Jiangsu 211166, China \\ 'Department of Nursing, The First Affiliated Hospital with Nanjing Medical University, Nanjing, Jiangsu 211166, China
}

Received: 24 November 2017; Accepted: 25 April 2018; Published: 20 December 2018

\begin{abstract}
Venous leg ulceration (VLU) is one of the complications of lower extremity venous reflux and reflux disorder of severe diseases, with many adverse effects on patient's work and life. Nowadays, more and more patients with VLU accept wound care in community setting. Clinical nurses generally take care of the patients based on their own experiences. Healing in VLU is an incredibly complex process, which puzzles even experts. The majority of general nurses do not have this level of expertise, especially those nurses serving in community. Function is the basis of nursing activities. Patients always show different clinical manifestations and selfcare abilities due to various function states, which cannot be reflected completely by the existing nursing practice. How to describe nursing practice standardized in order to demonstrate the effectiveness of interventions and facilitate interdisciplinary communication is another urgent problem. Therefore, the aim of this project is to develop an accurate nursing program based on function in chronic venous leg ulcers, which can both satisfy the needs of patients and promote nursing revolution.

Methods: This study will use International Classification of Functioning Disability and Health as a framework to choose suitable functions and to filter function classification standards of chronic venous leg ulcers through evidence-based systematic research. Nursing interventions related to VLU are selected based on Nursing Interventions Classification, adding other nursing activities by methods of evidence-based systematic review and clinical observation. Then, nursing interventions and function status are matched through steering committee. Finally, the Delphi survey method is adopted to make nursing program native and scientific.

Conclusions: This study is expected to be very significant and meaningful in using standardized nursing terminology. The nursing program established could better meet the needs of both patients with chronic venous leg ulcers and clinical nurses, promoting the development of wound specialist and standardized nursing language.
\end{abstract}

Keywords: accurate nursing program • venous leg ulceration • function status - nursing intervention classification • systematic review - Delphi survey •ICF・LUMT

(c) Shanxi Medical Periodical Press.

\section{Background}

Venous leg ulceration (VLU) is the most prevalent form of difficult-to-heal wounds worldwide. Healing process has

${ }^{\dagger}$ This study was supported by Innovative Research Team in Jiangsu Province (Suwei Kejiao [2016]22) and Jiangsu University Brand Professional Construction Project of Nursing (Sujiaogao [2015]11).

* Corresponding author.

E-mail: sryhlb@163.com (Z.-J.Gu).

2 Open Access. ๑ 2018 Yi-Fan Tang et al., published by Sciendo. (G) BY-Nc-ND This work is licensed under the Creative Commons Attribution NonCommercial-NoDerivatives 4.0 License. been reported to last at least $1-5$ years, some even last more than 10 years. ${ }^{1}$ Although about $1 \%$ of the population experiences a chronic venous leg ulcer, the prevalence in $>65$-year-old population is as high as $4 \%$. In addition, the prevalence rates of VLUs will permanently increase along with aging society. ${ }^{2}$ VLU is one of the 
complications of lower extremity venous reflux and reflux disorder of severe diseases. The ulcer has poor capacity of healing, remains unhealed for a long time, and recurs easily. ${ }^{3}$ Patients with VLUs are always confused by the chronic condition of local ulcers, such as loss of mobility and complex wound management, leading to inferior quality of work and life. ${ }^{3}$ The term "Marjolin's ulcer" is used to describe cancers arising from chronic wounds, especially venous insufficiency ulcers and pressure ulcers. The precancerous potential of chronic ulcers of lower limbs has been reported to result in amputation or death by several case reports. ${ }^{4,5}$ The principal etiology of the development of VLU is chronic venous insufficiency (CVI). CVI is a complex of symptoms and signs based on an inadequate venous return, which leads to longduration venous hypertension or venous obstruction and finally results in venous ulcers. ${ }^{6}$ In addition, such diseases are considered as risk factors for VLU, including arterial hypertension, diabetes mellitus, vasculitis, and cancer. As well as unhealthy lifestyle such as smoking, obesity, and sedentariness is noteworthy. ${ }^{2}$ Overall, the importance of integrated individualized intervention is emphasized.

In 2016, European Dermatological Forum (EDF) released a new guideline on the management of venous leg ulcers, which was based on the already existing EDF guideline on venous leg ulcers (2006). ${ }^{7}$ The treatments mentioned in the guideline were based on evidence in order to support the daily practice, including compression therapy, treatment of wounds, invasive treatment, oral medication, and after treatment lifestyle. However, nursing interventions or treatments described in the existing guidelines are not superexcellent, which are all from a certain way to guide clinical practice. Nursing practitioners cannot select suitable interventions from the guidelines to meet patients' individual needs according to present conditions. For compression therapy described in the guidelines, healing is faster with the use of compression than without; a high compression is better than a low compression, and multiple layers of a bandage are more effective than a single layer. There is no evidence that a particular type of compression therapy is optimal. Some studies reported that we can choose the type of compression according to clinical preferences, economy, and patient's compliance. ${ }^{8,9}$ About $15 \%-30 \%$ of the patients with VLUs have concomitant arterial dysfunction presenting with a reduced ankle-brachial pressure index (ABPI). ${ }^{10}$ Moderate compression is recommended for patients with arterial disease instead of high compression. ${ }^{11}$ In addition, it has been reported that the main side effects of compression therapy are dryness of skin (58.5\%) and itching $(32.7 \%) .{ }^{12}$ However, there is no solution recommended by the existing guideline.
Nowadays, wound care for patients with VLUs almost by nurse experiences. It has been reported that more and more patients with wounds need wound management in the community setting. ${ }^{13}$ The nursing activities for patients with VLUs in various institutions cannot reach unitary quality due to the limitations of skills and methods. The status quo of wound care has reported that the demand of advanced practice nurses exceeds supply. VLU is an incredibly complex process, which challenges even experts. Moreover, the majority of general nurses do not have this level of expertise, especially those nurses serving in community. ${ }^{14}$ There is loss of standardization and professionalism for the management of wound, including nursing assessment, diagnosis, and interventions.

It is a growing recognition that there is a need for a more accurate approach toward nursing interventions. Imitating the definition of precise medicine, accurate nursing means the right nursing interventions for right patients at the right time on the basis of comprehensive evaluation. The definition of accurate nursing intervention is unclear, which is not complete compared with personalized care. The Nursing Interventions Classification (NIC) is a system of nursing interventions classification and is related to the diagnosis classification of the North American Nursing Diagnosis Association International (NANDA-I). ${ }^{15}$ When doing nursing assessment, diagnosis and interventions, clinical nurses always focus on patient's function status. The International Classification of Functioning, Disability and Health (ICF) provides a comprehensive, universally accepted framework for the description of health-related functioning. ${ }^{16}$ The combination of NIC and ICF can describe the standardized nursing interventions, which are most suitable for patients' function status, as well as to facilitate interdisciplinary communication and penetration. ${ }^{17}$ In summary, accurate care is based on comprehensive evaluation to provide the most appropriate nursing intervention for patients' certain function states in the framework of standardized nursing terminology. Nursing care according to function classification has been reported to be practiced in several diseases, including acute leukemia, stroke, and chronic obstructive pulmonary disease. The advantage of it has been proved to be more important for providing individual nursing care. ${ }^{18-20}$

There is demand to develop an accurate nursing program based on function for venous leg ulcers. Involvement of specialized subject experts in developing the program through the Delphi consensus project is likely to accelerate its uptake. By providing a nursing program for clinical nurses to follow, which can lead to faster wound healing, a reduction in complications, shorter patient stay, and finally improved quality of care that results in reduced costs are observed..$^{13}$ The use 
of standardized nursing terminology will also promote nursing practice and drive nursing revolution.

\section{Aim}

The aim of this project is to develop a core function with VLU, function classification standard, and accurate nursing program in order to build clinical practice involving patients with VLUs.

\section{Design/methodology}

The study was conducted by a research group, which consists of one nursing director, one enterostomal therapist, and one nursing postgraduate. There are three steps in this study. The first step is preliminary questionnaire formulation. ICF is used as the framework to choose suitable function, and function classification standards of chronic venous leg ulcers are filtered through evidence-based systematic research. Nursing interventions are selected from NIC, with the addition of other nursing activities by methods of evidence-based systematic review and tracking observation. The second step is to modify and adjust nursing program, which is completed by first step undergoing steering committee. Finally, nursing interventions and VLU function status perfectly match by several rounds of Delphi survey. The final version can surely meet the local cultural background.

\subsection{Related functions and function classification standards of chronic venous leg ulcers}

\subsubsection{Skin function (b810-b849)}

Local ulcer wound is the principal and main symptom of venous leg ulcer. Accurate wound assessment is essential for the appropriate and realistic planning of goals and interventions for patients with wounds. ${ }^{21}$ Physical examination is considered extremely important for local wound treatment. ${ }^{7}$ The Leg Ulcer Measurement Tool (LUMT) is the first instrument developed to evaluate and track changes in leg ulcer appearance over time, which represents a novel assessment tool specifically designed and validated for chronic leg ulcers. ${ }^{22}$ The tool is easy to master, and it only takes 3 minutes to complete the form by a clinical nurse after training. LUMT contains a number of systematic and must-be-considered components for chronic leg ulcers (Table 1).

\begin{tabular}{|c|c|c|c|c|c|}
\hline Assessment & 0 & 1 & 2 & 3 & 4 \\
\hline A1.Exudate type & None & Serosanguineous & Serous & Seropurulent & Purulent \\
\hline A2. Exudate amount & None & Scant & Small & Moderate & Copious \\
\hline A3.Size (length $\times$ width) & Healed & $<2.5 \mathrm{~cm}^{2}$ & $2.5-5.0 \mathrm{~cm}^{2}$ & $5.1-10.0 \mathrm{~cm}^{2}$ & $\geq 10.1 \mathrm{~cm}^{2}$ \\
\hline A4.Depth & Healed & Partial thickness skin loss & Full thickness & $\begin{array}{l}\text { Tendon/joint capsule } \\
\text { visible }\end{array}$ & Probes to bone \\
\hline $\begin{array}{l}\text { A5.Undermining } \\
\text { (greatest at_o'clock) }\end{array}$ & $0 \mathrm{~cm}$ & $>0-0.4 \mathrm{~cm}$ & $>0.4-0.9 \mathrm{~cm}$ & $>0.9-1.4 \mathrm{~cm}$ & $>1.5 \mathrm{~cm}$ \\
\hline A6. Necrotic tissue type & None & Loose white to yellow slough & $\begin{array}{l}\text { Attached white to } \\
\text { yellow slough or fibrin }\end{array}$ & Soft grey to black eschar & $\begin{array}{l}\text { Hard dry black } \\
\text { eschar }\end{array}$ \\
\hline $\begin{array}{l}\text { A7. Necrotic tissue } \\
\text { amount }\end{array}$ & $\begin{array}{l}\text { None } \\
\text { visible }\end{array}$ & $\begin{array}{l}1-25 \% \text { of wound bed } \\
\text { covered }\end{array}$ & $\begin{array}{l}26-50 \% \text { of wound bed } \\
\text { covered }\end{array}$ & $\begin{array}{l}51-75 \% \text { of wound bed } \\
\text { covered }\end{array}$ & $\begin{array}{l}76-100 \% \text { of wound } \\
\text { bed covered }\end{array}$ \\
\hline $\begin{array}{l}\text { A8.Granulation tissue } \\
\text { type }\end{array}$ & Healed & Bright beefy red & Dusky pink & Pale & Absent \\
\hline A10.Edges & Healed & $\begin{array}{l}\geq 50 \% \text { advancing border } \\
\text { of epithelium or indistinct } \\
\text { borders }\end{array}$ & $\begin{array}{l}<50 \% \text { advancing } \\
\text { border of epithelium }\end{array}$ & $\begin{array}{l}\text { Attached, no advancing } \\
\text { border of epithelium }\end{array}$ & $\begin{array}{l}\text { Unattached or } \\
\text { undermined }\end{array}$ \\
\hline $\begin{array}{l}\text { A11.Periulcer skin } \\
\text { viability }\end{array}$ & None & One only & Two or three & Four or five & Six or more factors \\
\hline A12.Leg edema type & None & Nonpitting or firmness & Pitting & $\begin{array}{l}\text { Fibrosis or } \\
\text { lipodermatosclerosis }\end{array}$ & Indurated \\
\hline A13.Leg edema location & None & Localized periulcer & Foot, including ankle & To mid-calf & To knee \\
\hline $\begin{array}{l}\text { A14.Assessment of } \\
\text { bioburden }\end{array}$ & Healed & Lightly colonized & Heavily colonized & Localized infection & Systemic infection \\
\hline B1.Pain amount & None & $>0-2$ & $>2-4$ & $>4-7$ & $>7$ \\
\hline B2.Pain frequency & None & Occasional & Position dependent & Constant & Disturbs sleep \\
\hline B3.Quality of life & Delighted & Satisfied & Mixed & Dissatisfied & Terrible \\
\hline
\end{tabular}

Table 1. Leg ulcer measurement tool.

Note: A11: factors affected include callus, dermatitis (pale), maceration, induration, erythema (bright red), purple blanchable, purple nonblanchable, and skin dehydration B1 and B2: it relates to the leg ulcer and experience in the last 24 hours. B3: it relates to the leg ulcer. 


\subsubsection{Vessel function (b415)}

ABPI can assess peripheral arterial blood supply and assist with the differential diagnosis of leg ulcers. ${ }^{23}$ Doppler sonography is a simple, cheap, and practical screening technique for measuring the ABPI. Compression therapy could potentially restrict arterial perfusion, and so it is crucially important to be determined before pressure therapy for security purpose. Many studies have reported that $A B P I$ can be used to choose the strength of compression and recommend "reduced" compression in patients with arterial dysfunction showing a lower ABIP.11 Furlong ${ }^{24}$ has reported the need of precise national guidelines with regard to APBI review for patients under compression therapy, which can make compression therapy more scientific and logical (Tables 2 and 3)..$^{25,26}$

\subsection{Nursing intervention classification}

Research identifies the following top 10 NANDA-I nursing diagnoses of patients with VLUs: (1) activity intolerance, (2) knowledge deficient, (3) mobility: physical, impaired, (4) noncompliance, (5) pain, acute, (6) pain, chronic, (7) skin integrity, impaired, (8) tissue integrity, impaired, (9) injury, risk for, and (10) skin integrity, risk for impaired. Through NANDA-I, NIC, NOC, and NNN linkages, 23 nursing interventions were selected, which were the most frequently used from NIC as follows: activity therapy, exercise promotion, exercise therapy: ambulation, teaching: procedure/treatment, teaching: disease, health education, pain management, pressure management, pressure ulcer care,

\begin{tabular}{llllll}
\hline ABPI & $>1.3$ & $0.90-1.30$ & $0.81-0.89$ & $0.51-0.8$ & $<0.5$ \\
\hline \hline Result & $\begin{array}{l}\text { Abnormal } \\
\text { vessel } \\
\text { hardening }\end{array}$ & Normal & Abnormal & $\begin{array}{l}\text { Significant } \\
\text { ischemia }\end{array}$ & $\begin{array}{l}\text { Severe } \\
\text { ischemia }\end{array}$ \\
\hline
\end{tabular}

Table 2. Ankle-brachial pressure index (ABPI). medication management, thrombosis care: peripheral vessel, thrombosis protection, air pressure tourniquet protection, medication administration, medication administration: skin, skin surveillance, skin care: topical treatments, wound care, infection control, infection protection, tumble protection, security enhancement, and transfer treatments.

\subsection{Supplement for nursing intervention}

\subsubsection{Systematic review}

(1) Criteria for consideration of the included studies

The studies that attempted to investigate which nursing interventions can shorten the healing process of patients with VLUs or promote better life were included. The focus will be on nursing activities such as compression therapy, exercise therapy, lifestyle intervention, debridement, and local wound measurement. The types of studies included are as follows: literature reviews with/without meta-analysis, randomized controlled trials, controlled trials, case series, prospective cohorts, and qualitative research.

\section{(2) Exclusion criteria}

Papers or articles (both experimental and exploratory) that only consider the treatment or decision made by doctors without nursing intervention or decision were excluded. The research studies that included pregnant females, patients with leg ulceration caused by diseases other than venous disease, and patients with active cellulitis were also excluded.

\section{(3) Search methods}

The databases such as Medline (from 1950), Embase (from 1947), PubMed (from 1966), Web of Science

\begin{tabular}{lll}
\hline Low risk & Medium risk & High risk \\
\hline \hline $\begin{array}{l}\text { All the factors below must be present } \\
\text { Review annually }\end{array}$ & $\begin{array}{l}\text { Ankle-brachial pressure index (ABIP) }=0.81-1.3 \\
\text { With accompanying score of 2 or more } \\
\text { Review every } 6 \text { months }\end{array}$ & $\begin{array}{l}\text { If any one risk factor below is present } \\
\text { Review every } 3 \text { months }\end{array}$ \\
ABIP 0.81-1.3 & Smoker & ABIP $<0.81-1.3$ \\
Mobile & Age, 70+ & Diabetes \\
Good cognitive ability & Cardiac disease & Immobile/limited mobility \\
No complications & Cerebral vascular accident & Small vessel disease (i.e., diabetes) \\
$\begin{array}{l}\text { Patient has knowledge of early symptoms } \\
\text { of peripheral arterial disease (PAD) }\end{array}$ & Transient ischemic attack & Angioplasty/bypass (lower limbs) \\
$\begin{array}{l}\text { Patient has contact numbers if there } \\
\text { are any concerns }\end{array}$ & Comorbidities (learning needs, cognitive & Inflammatory conditions, i.e., rheumatoid arthritis \\
& impairment, drug/alcohol dependency, and & Systematic lupus erythematous \\
Intermittent claudication \\
\end{tabular}

Table 3. Peripheral arterial risk chart. 
(from 2005), and CINAHL (from 1981) were searched for literature, and in addition, CENTRAL and Cochrane Methodology Register were searched for experimental studies. A search for additional studies was carried out by checking the references of the included studies.

\section{(4) Eligibility of studies}

Citations identified through searches were independently evaluated by two reviewers (from research group). Full-text papers were obtained for those studies that are considered potentially relevant after citation evaluation, and further assessment was made for inclusion. Any papers not meeting inclusion criteria were excluded. The included full-text papers were assessed independently by two reviewers in detail. Any disagreement was judged by a third reviewer.

\section{(5) Data extraction}

Information from the primary studies was selected independently by two reviewers and reviewed to evaluate the agreement that all outcomes have been identified. Some included papers' information was extracted and summarized from each study as follows: first author, year and journal of publication, country, study type, study aim, and host study context (nursing intervention outcomes). Specific details on the nursing intervention are most important such as time, period, implementer, place, intensity, and equipment.

\subsubsection{Guideline review}

National Guideline Clearinghouse, Registered Nurses' Association of Ontario, Scottish Intercollegiate Guidelines Network, and Best Practice database were searched for guidelines on venous leg ulcers or venous leg diseases. Eligibility and data extraction of the studies are similar to the method of systematic literature review.

\subsubsection{Observation method}

Nurses or caregivers who make nursing intervention for patients with VLUs in Department of Vascular Surgery and Clinic Wound Department were followed by a researcher. All their activities to take care of VLUs will be recorded timely. The observer records the primary task or activity at the time of observation with pen onto a preset data collection form. Then, the researcher attempts to match the activities with interventions for VLUs described in Section 3.2. While the activities failing the match will be record in detail to supply for nursing intervention in this research. The researcher, who was trained by work sampling technique, observed all nurses or assistants on duty for several days, 8 hours for each day (8:00-16:00), and recorded a snapshot of nursing intervention for VLUs at 10-minute intervals until data saturation. ${ }^{27}$

\subsection{Steering committee}

A steering committee was formed to complete an accurate nursing program. Experts are from various disciplines (rehabilitation, wound care, dermatology, and nursing research), who have been engaged in clinical nursing work for at least 10 years and volunteer to take part in this research. The committee was established by faceto-face meetings for about $2-4$ hours. The target is to match the nursing activities and interventions that come from NIC, methods of systemic review, guideline review, and clinical observation with patients' function status and make suggestion for revision, addition, or deletion if necessary. Any disagreement will be discussed together. Finally, the research group makes preferable accurate nursing program for the next Delphi survey.

\subsection{Delphi survey}

The Delphi survey was formed to obtain consensus about how logical and scientific the match between patients' function grades with VLUs and nursing interventions. Experts who have been engaged in clinical care for at least 10 years, own rich experience both in nursing research and wound care, and volunteer to take part in the study were included. Only those who complete round 1 and have a certain understanding of this subject were invited to participate in the next round. We used a purposive and snowball sampling strategy and aim for the recommended minimum sample size of 20 .

Survey was conducted for at least two rounds until experts' opinion arrives at a general agreement. Each survey round was online for 1 week, and the next round was sent after a 2-week hiatus in order to avoid experts' memory bias. In every round, participants were asked to rank the importance of nursing interventions for relevant function status in the grid by rating each intervention on a 5-point Likert rating scale (1-5), from 1: strongly disagree to 5: strongly agree. They will also be requested to provide suggestion for any revision, addition, or deletion. After each round, the result of consultation was collated and sent to participants in the next round. Each survey spends 30-50 minutes to complete with request for participants to answer in the order and review answers before final submission.

After each round, questionnaires are collected, and two researchers from the research group will build 
database using software Excel 2007 and Statistic Package for Social Science 20.0 to calculate mean rank for each activity and assess consensus for each list within each round using Kendall's W. The activity that has a mean score of $>3.5$ and $70 \%$ of the experts scoring 4 or above was included. The value of $\mathrm{W}$ ranges from 0 to 1 , with 0.7 or greater indicating satisfactory agreement, and we would consider the ranking phase completed.

\section{Ethical considerations}

Research ethics committee approval was obtained from ethics review committee (April 2017). All individual participation will be voluntarily sought following the presentation of written information about the theoretical purposes of the study and informed of their right to decline to provide specific information or to terminate participation at any phase in the study without detriment. Participants' information will be kept anonymous and confidential.

\section{Validity and reliability}

Several steps were carried out to assure the validity and reliability of this study. First, a research group carried out the whole project, in which some phases were implemented independently (e.g. filtering literature). Second, the experts of steering committee and Delphi survey are all major-related and excellent in the field of wound care or nursing research. Third, the abovementioned appraisal tools were proved to be effective and valid in previous studies. Finally, the accurate nursing program was assessed through the Delphi survey, which is a versatile and mature tool to collect opinions widely.

\section{Discussion}

At present, Gu Zejuan team from China is the only research team to develop an accurate nursing program based on patients' function with a disease, using NIC and ICF as frameworks. Studies have already been carried out to verify the clinical effect of accurate nursing program on cardiac function, neuromuscular function, pulmonary ventilation function, and blood function. ${ }^{18-20}$

In the course of study, we found that it was not enough to use standard nursing terminologies as a research framework. There is an unavoidable topic to develop and apply accurate nursing program, which is suitable for clinical practice and medicine technology in various countries. The Delphi method is used in this study to make the nursing program, which is made by NIC, method of literature review, and clinical observation, fit to related function states, conforming to Chinese local background. Implementation of Delphi survey is scientific and reasonable.

The characteristics of this study also contain the application of nursing program. Clinical practitioners make nursing assessment of patients using sensitive and specific LUMT. It can provide patients with best interventions and cares. The next time is both following assessment and feedback evaluation. For consistency, the measurement cycle of ABIP should also be supported by evidence. ${ }^{24}$

The aim of this study is to develop an accurate nursing program based on function for chronic venous leg ulcers. The template should be generalizable to build an accurate nursing program across other acute or chronic wounds, including diabetic foot, bedsores, operative wound, and so on. From single diseases to specialized diseases, our future development must focus on the combination of standard nursing terminologies and continuous function assessment. In the future, we would apply information system in the accurate nursing program. ${ }^{28}$ Nurses would only spend several minutes to input the sign of patients' function states, and then the information terminal will output the corresponding interventions and activities from the system, which is efficient and time-saving for nursing.

\section{Trial status}

Overall, the protocol does not report a trial but a development of a function and function classification standard set for use in future studies of accurate nursing program to guide clinical practice. Next step is to make a supplement for nursing activities and interventions through literature review. Detailed information about nursing activities and intervention for VULs for different parts will be collected.

\section{Limitations}

The program is only carried out in China. It is expected that the following development will be further carried out in different countries. Core functions about VLU from ICF have been mentioned, but others are still unclear. In general, few methodological guidelines are available, resulting in the absence of reference standard to develop functions and function classification standards.

\section{Conflicts of interest}

All contributing authors declare no conflicts of interest. 


\section{References}

1. Vowden KR, Vowden P. The prevalence, management and outcome for patients with lower limb ulceration identified in a wound care survey within one English health care district. J Tissue Viability. 2009;18:13-19.

2. Green J, Jester R, McKinley R, Pooler A. The impact of chronic venous leg ulcers: A systematic review. J Wound Care. 2014;23:601-612.

3. O'Brien JA, Finlayson KJ, Kerr G, Edwards HE. Testing the effectiveness of a self-efficacy based exercise intervention for adults with venous leg ulcers: Protocol of a randomised controlled trial. BMC Dermatol. 2014; 14: 16.

4. Enoch S, Lefemine V. Recalcitrant leg ulcers and the risk of cancerous degeneration. J Endodontics. 2007; 14:125-127.

5. Onesti MG, Fino P, Fioramonti P, Amorosi V, Scuderi N. Ten years of experience in chronic ulcers and malignant transformation. Int Wound $\mathrm{J}$. 2015;12:447-450.

6. de Almeida Medeiros AB, de Queiroz Frazão CM, de Sá Tinôco JD, Nunes de Paiva Md, de Oliveira Lopes MV, Brandão de Carvalho Lira AL. Venous ulcer: risk factors and the nursing outcomes classification. Invest Educ Enferm. 2014;32:252-259.

7. Neumann M. Evidence-based (S3) Guidelines for diagnostics and treatment of venous leg ulcers answer to Dr Bertolini. J Eur Acad Dermatol Venereol. 2017;31:e386.

8. Nelson EA, Harrison MB, Canadian Bandage Trial Team. Different context, different results: venous ulcer healing and the use of two high-compression technologies. J Clin Nurs. 2014;23:768-773.

9. O'Meara S, Tierney J, Cullum N, et al. Four-layer compression bandage compared with short-stretch bandage for venous leg ulcers: A meta-analysis of randomised controlled trials using individual patient data. Wound Repair and Regeneration. 2007;15:A128-A128.

10. Mosti G, labichella ML, Partsch H. Compression therapy in mixed ulcers increases venous output and arterial perfusion. J Vasc Surg. 2012;55:122-128.

11. Mosti G, Cavezzi A, Massimetti G, Partsch H. Recalcitrant venous leg ulcers may heal by outpatient treatment of venous disease even in the presence of concomitant arterial occlusive disease. European J Vascular \& Endovascular Surg. 2016;52:385-391.

12. Reich-Schupke S, Murmann F, Altmeyer $P$, Stücker M. Quality of life and patients' view of compression therapy. Int Angiol. 2009;28:385-393.
13. Greatrex-White S, Moxey H. Wound assessment tools and nurses' needs: An evaluation study. Int Wound J. 2015;12:293-301.

14. Fletcher J. Optimising wound care in the UK and Ireland: a best practice statement. 2008.

15. Head BJ, Scherb CA, Reed D, et al. Nursing diagnoses, interventions, and patient outcomes for hospitalized older adults with pneumonia. Res Gerontol Nurs. 2011;4:95-105.

16. Bölte S, de Schipper E, Robison JE, et al. Classification of functioning and impairment: yhe development of icf core sets for autism spectrum disorder. Autism Res. 2014;7:167-172.

17. Van Achterberg T, Holleman G, Heijnen-Kaales Y, et al. Using a multidisciplinary classification in nursing: the international classification of functioning disability and health. J Adv Nurs. 2005;49:432-441.

18. Li R, Gu ZJ, Wang X, Yu L. Observation on the effect of nursing scheme based on functional state in early hemiplegia patients with stroke. J Nurs. 2016;23:45-49 (in Chinese).

19. Zhou Y, Gu ZJ, Jiang XM, Wei XL. Application of functioning-driven nursing plans in acute leukemia patients. J Nurs Sci. 2016;3:27-30 (in Chinese).

20. Wang L, Liu KY, Gu ZJ, Zhu JX, Chen CF. Application of function-based nursing program in patients with pulmonary ventilation dysfunction. J Nurs Sci. 2015;5:19-22 (in Chinese).

21. Pillen $\mathrm{H}$, Miller $\mathrm{M}$, Thomas $\mathrm{J}$, et al. Assessment of wound healing: validity, reliability, and sensitivity of available instruments. Wound Pract Res. 2009;17:208-217.

22. Woodbury MG, Houghton PE, Campbell KE, Keast $\mathrm{DH}$. Development, validity, reliability, and responsiveness of a new leg ulcer measurement tool. Adv Skin Wound Care. 2004;17:187-196.

23. Group HMN. Recommended practice: Anklebrachial pressure index (ABPI) using hand-held doppler ultrasound. Wound Pract Res. 2014;22.

24. Furlong W. Recommended frequency of abpi review for patients wearing compression hosiery. Br J Nurs. 2015;24 Suppl 20:S18-S23.

25. Woo KY, Cowie BG. Understanding compression for venous leg ulcers. Nursing. 2013;43:66-68.

26. Nazarko L. Simplifying the management of venous leg ulcers: Choosing appropriate and acceptable compression therapy. $\mathrm{Br} J$ Community Nurs. 2017;22(Sup6):S6-S12.

27. Blay N, Duffield CM, Gallagher R, Roche M. Methodological integrative review of the work sampling 
technique used in nursing workload research. $J$ Adv Nurs. 2014;70:2434-2449.

28. Dharmarajan B, Gangadharan K. Applying technology acceptance (TAM) model to determine the acceptance of nursing information system (NIS) for computer generated nursing care plan among nurses. Int $J$ Computer Trends Tech. 2013;4:841-864.

How to cite this article: Tang Y-F, Hu X-Y, Zhao J, Gu Z-J, Xing S-S, Wang W-Y. Development of an accurate nursing program based on function in chronic venous leg ulcers: protocol for matching function status with nursing interventions using the Delphi survey. Front Nurs. 2018; 4: 265-272. https://doi.org/10.1515/fon-2018-0036. 